\title{
Introduction to the Communication and Information Systems Technology for Crisis and Disaster Management minitrack 2017
}

\author{
Julie Dugdale \\ University Grenoble-Alps, \\ CNRS \\ Grenoble Informatics Lab (LIG) \\ University of Agder \\ dugdale@imag.fr
}

\author{
Jose J Gonzalez \\ University of Agder \\ Centre for Integrated Emergency \\ Management \\ josejg@uia.no
}

\author{
Murray Turoff \\ Information Systems Department \\ New Jersey Institute of Technology \\ murray.turoff@gmail.com
}

According to the United Nations Office for Disaster Risk Reduction (UNISDR) the economic cost of disasters from 2005 to 2014 has cost our society 1.4 trillion dollars. Even in the United States the majority of the costs are not covered by any form of insurance. Moreover, the human impact has been staggering with 1.7 billion people being directly affected and 0.7 million people losing their lives to disasters. The rising impacts are clear and the challenge of designing communication and information systems technology for disaster management has never been greater. Research into this area has been undertaken for several years now. In 2013 the first minitrack dealing with communication and information systems technology for crisis and disaster management was introduced at HICSS. At the $50^{\text {th }}$ anniversary of HICSS it is a good time to reflect on the remarkable and rapid advances that have been made in our domain. The first minitrack paved the way for examining the critical issues in our field with looking at data collection to aid better management, lessons learnt from previous disasters, and decision support systems. These topics are still hotbeds of research. However since the inception of this minitrack we have seen the other areas emerge, such as: the increase in use of mobile devices and social media for self-organisation in the face of a crisis and for two-way reporting between citizens to crisis managers; the affect of new technologies, such as advances in wireless networks, communication devices and applications that support crisis management; the increase in frameworks, platforms and approaches for studying and developing information systems for crisis management. Looking ahead, new challenges are on the horizon, we are moving towards a more holistic view of crisis and disaster management so as to include an increasing interest in agility and resilience as defining characteristics of good management. Emerging themes are appearing, such as the rising interest in disaster e-Health, UAVs and disaster robotics, as well as ethical legal and social issues associated with disaster management.

There are 10 papers in this minitrack, divided into three sessions. The first session brings together papers concerning social media and information needs. Social media systems offer the promise of potentially supplying critical, up to date information that could aid emergency managers in setting their response priorities and delivering aid. However, there are many barriers currently preventing optimal use of social media for this purpose. The first paper by Runqing Rao, Linda Plotnick and Roxanne Hiltz, "Supporting the Use of Social media by Emergency Managers: Software tools to Overcome Information Overload" is based on data from the third in a series of studies exploring the issues impeding optimal use of social media by county-level emergency managers in the U.S. The study focuses on potential software solutions to the overload and trust issues, which our research shows are major barriers. The overarching goal for this research is to better understand the hesitance of government to use social media, especially for data collection, and to build a communication bridge between potential users and the technologists who build social media platforms and data processors so the full potential of social media use can be reached.

The second paper in this session by Rajib Subba and Tung Bui titled "Online Convergence Behavior, Social Media Communications and Crisis Response: An Empirical Study of the 2015 Nepal Earthquake Police Twitter Project" looks at supporting communication exchange between crisis managers and the community. Crisis management narratives traditionally revolve around emergency response authorities and focus on logistics and human resource management. However, during the process, survivors' actual needs are often over looked. This paper demonstrates that social media could be used as an effective communication and collaboration platform between the public authority and the general public, making it easier for the former to serve the community. 
Moreover the preliminary findings of the paper indicate that institutional elements do mediate social media activities of the authorities.

In the third paper of this session No-San Lee, Stefan Hirschmeier, Sven Müller, Lars Jürgen Luz look at "Enablers in Crisis Information Management: A Literature Review". Due to situations of emergency at major events (e.g. terrorist attack or natural hazard during a festival) relief units have to make decisions dynamically on the basis of a large amount of different information. In this context social media can provide decision-critical information and should be part of a modern communication concept. Smartphones as common mobile devices with multi-sensory features are enhancing the traditional ways of communication and are gaining more and more importance to relief organizations in crisis situations and catastrophes. Both information and communication technologies (ICT) have the ability to enable actors to react more effectively and more efficiently when managing crises. On the one hand the identification of ICT-enabled potentials for relief organizations are of great interest. On the other hand the identification and evaluation of ICT-enabled impact factors on the phases and processes of crisis management must be considered. One of the main challenges in this research is to identify enablers in order to integrate both views into a crisis information and communication framework with scope on improving and developing ICT services and ICT-supported communication concepts for relief organizations.

The last and final paper in the social media and information needs by Robert Power and Justin Kibell describes "The Social Media Intelligence Analyst for Emergency Management". The community is now highly connected, digitally literate and equipped with mobile devices. People expect real-time information to be readily available on demand, such as online banking, Google/Apple maps, weather forecasts, public transport planners and so on. This community expectation is expanding into the way governments and citizens interact: people expect to be able to engage with government services using social channels. This is also the case during emergency events. While emergency management organisations utilise data from various sources to help with prediction, management and forensic analysis of disasters and crisis, with the aim of improving situation awareness and crisis management, crowd sourced content from the community has to date largely been viewed with caution. This is mainly due to issues of reliably finding relevant information, establishing its veracity and trustworthiness and the lack of time available during critical emergency events. The paper by Robert Power and Justin Kibell addresses these issues by describing how the new operational role of the Social Media Intelligence Analyst utilises social media to support decision making for emergency management in a State Control Centre in Australia.

The second session of paper concerns methodologies and technologies. The first paper by Aladdin Shamoug and Radmila Juric is titled "Software Tool for Semantic Resources Allocation in Humanitarian Crises". This tool enables continuous support in resource allocation from day 1 of humanitarian crises and supports decision making by collecting and interpreting semantics of an environment where humanitarian response is required. The emphasis is on understanding the meaning of available and constantly changing information in humanitarian crisis. The tool also allows the input of various types of information from heterogeneous existing and persistent repositories and gives provision for accepting data from media and social media. One of the most important aspect of the tool is that its computational model, which assists in decision making, is based on SWRL enabled OWL ontologies, which allow the interpretation of collected information and reasoning upon it for semantic resource allocation.

The second paper in this session is by Frederick Benaben, Aurelie Montarnal, Sebastien Truptil, Matthieu Lauras, Audrey Fertier, Nicolas Salatge, Sebastien Rebiere and concerns "A conceptual framework and a suite of tools to support crisis management". This work deals with information systems to deal with agile management of local, regional or national crises. The presented model-driven suite of software tools is dedicated to (i) collect data to constantly model the crisis situation (including the responders and their capabilities, the context and its specificities, the crisis itself and the associated objectives), (ii) deduce and orchestrate the appropriate optimized collaborative behaviour schema (based on doctrines and plans elements) and (iii) maintain and adapt this collaborative behaviour by detecting any disruption or unexpected event (and re-deducing the appropriate behaviour schema). The underlying knowledge management is based on a crisis management metamodel and on the associated management rules (to deal with the available data to deduce, interpret, detect according to this metamodel). In addition, The IOSUITE tools are available online.

In their paper "Comparing Agent Architectures in Social Simulation: BDI Agents versus Finite-State Machines", the authors Carole Adam, Patrick Taillandier, and Julie Dugdale address an important challenge in Agent-Based Social Simulation when dealing with crisis situations, which is finding the right level of complexity for the agent architecture. Complex 
patterns of behaviour can emerge from very simple architectures, but it is sometimes necessary to use more realistic architectures to accurately model social human behaviour. Many simulations exist that are based on different underlying architectures, and it appears important to be able to compare them with each other. Our paper is an attempt at comparing two models of human behaviour in bushfires, one using a simple finite-state machine, the other one using a complex BDI architecture.

The last and final session concerns papers devoted to specific case studies and education. The first paper in this session is by Yang Ishigaki, Yutaka Matsuno, Koichi Bando, and Kenji Tanaka and concerns "Wisdom of Crowds for Reliable Discussion and Need Finding: A Case Study of Information Sharing Regarding Radiation after the Fukushima Nuclear Disaster". In crisis communication, social media is a useful tool for grasping the situation and understanding the needs in affected areas; however, it remains problematic in terms of reliability assurance and the ability to summarize posts. The authors of this paper facilitated the use of an inclusive Facebook community for radiation monitoring established by volunteer experts and normal users, soon after the Fukushima Nuclear Disaster. Interaction through this social media led to credible discussions and enabled dose verification among users. They also developed a Web service called Crowd Talks to summarize and visualize discussions within the Facebook community. The research discusses the application of Crowd Talks to the Facebook community in order to grasp a rough idea of the potential needs of and/or information required by users, especially those living in the affected areas.

Education is at the heart of good crisis management. The paper by Murray Turoff, Victor Banuls Silvera, and Lili Yang "A Review of Qualitative Comments on a proposed Master's degree in Emergency Management" reflects a long-term effort to improve the understanding in Emergency Management of the critical relationship to the design of supportive Information Systems. Associated with this has been the design of a degree program and associated courses. Ultimately, it led to a series of papers on education for Emergency Management in this area. The latest one is in this mini-track and focuses on the qualitative results of a survey of both practitioners and academics on this topic.

The final paper of this session looks at risks and emergency management from a small businesses point of view. Small businesses need to plan and react to business interruptions for which they may have little preparation. Essential steps in creating an emergency preparedness plan are to determine the risks that the business may face, and the mitigating actions that can be taken to lessen the potential impacts of these risks. The study that is reported in the paper "A Risk Scenario for Small Businesses in Hurricane Sandy Type Disasters" by Arthur Hendela, Murray Turoff, Starr Roxanne Hiltz, and Jerry Fjermestad, is based on Delphi surveys of over 50 small businesses affected by Hurricane Sandy. Using a Delphi approach followed by Cross Impact Analysis and Interpretive Structural Modelling the paper tries to determine the risk interactions and the highest valued scenario for which to prepare. Somewhat surprisingly, the highest ranked risk is loss of business reputation. The research method then combines individual events into scenarios that represent the most concentrated use of limited budgets for mitigating small business interruption. 